\title{
Neonatal subcutaneous fat necrosis with hypercalcemia treatment using calcitonin
}

\author{
Khouloud A. Alsofyani, MD.
}

\begin{abstract}

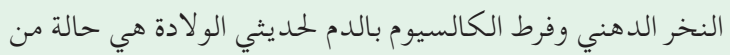

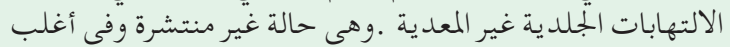

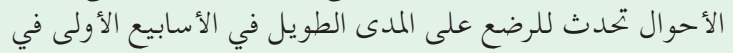

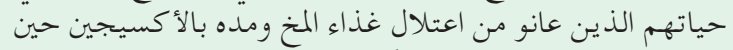

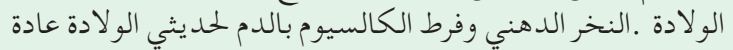

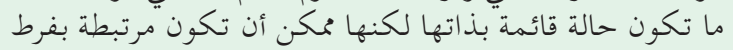

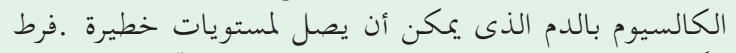

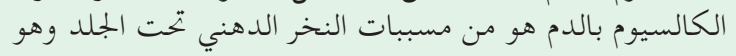

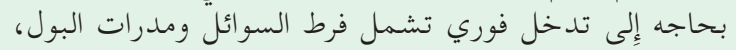

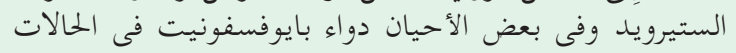

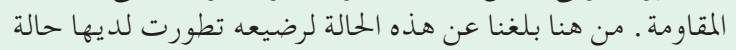

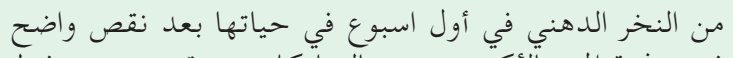

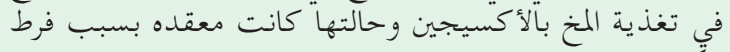

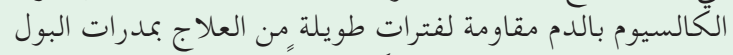

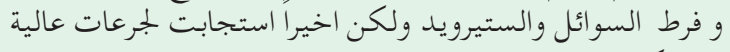

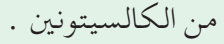

Subcutaneous fat necrosis of newborn is a form of noninfectious panniculitis. It is an uncommon condition and mostly occurs in full-term infants within first weeks of life that have had a significant condition such as hypoxic-ischemic encephalopathy at the time of birth. Subcutaneous fat necrosis of newborn is usually a self-limiting condition but may be associated with hypercalcemia, which may lead to life threatening levels. Hypercalcemia is a significant reason of subcutaneous fat necrosis and needs an intervention involving hyperhydration, calcitonin, diuretics, steroids and sometimes bisphosphonate in resistant cases. Here, we have reported the case of a term infant who developed extensive subcutaneous fat necrosis in the first week of life after significant perinatal hypoxic injury. Her condition was complicated by hypercalcemia resistant to prolonged medical treatment with hyperhydration, diuretic and steroid but eventually the infant responded to high doses of calcitonin.

Saudi Med J 2018; Vol. 39 (6): 622-626 doi: 10.15537/smj.2018.6.22025

\begin{abstract}
From the Department of Pediatrics, King Abdulaziz University Hospital, Jeddah, Kingdom of Saudi Arabia.
\end{abstract}

Received 23rd January 2018. Accepted 23rd May 2018.

Address correspondence and reprint request to: Dr. Khouloud $A$. Alsofyani, Department of Pediatric, Section of Pediatric Critical Care, King Abdulaziz University Hospital, Jeddah, Kingdom of Saudi Arabia.E-mail:alsofyani.drk@yahoo.com

Subcutaneous fat necrosis of the newborn (SCFN) is $\checkmark$ a rare disorder with multiple and varied etiologies. Subcutaneous fat necrosis of the newborn is characterized by erythematous plaques and nodules which eventually develops in calcification. It affects faces, backs, buttocks or shoulders of the term or post-term newborns. These nodules may appear in isolated or clustered form and are hard and painful. This disease generally develops within the first 6 weeks of life. Although the exact cause of SCFN is unknown, but certain maternal factors such as pre-eclampsia, gestational diabetes, use of calcium-channel blockers or cocaine by the mother, umbilical cord prolapses, Rh incompatibility, obstetric trauma, localized tissue hypoxia and neonatal asphyxia are linked to being predisposing for the disease onset. The occurrence of SCFN is generally linked with neonatal asphyxia. ${ }^{1,2}$ Despite being a benign condition, SCFN is known to be associated with disorders such as hypoglycemia, thrombocytopenia, hypercalcemia and hypertriglyceridemia. It is a temporary, autoresolving condition, still, the early diagnosis of SCFN is necessary for proper management and treatment. If left unattended, it may further lead to many complications that can have severe consequences at a later stage. The most significant and major complication of SCFN is hypercalcemia, which may prove to be life threatening. Severe increase in calcium levels might lead to nephrocalcinosis with a progressive reduction in renal function, calcification of skin, gastric mucosa and myocardium. Hypercalcemia might also lead to increased prostaglandin activity that will cause osteoclast activation, the release of calcium from necrotic fat cells and increased 1,25-dihydroxy vitamin $\mathrm{D}$ production 
which in turn causes increased bone turnover. ${ }^{3}$ Even after recovering from the condition of SCFN, many babies are left with cutaneous atrophy in the regions where there was the presence of lesions.

There are only few studies and reports on epidemiologic data of SCFN. Despite its low prevalence, both neonatologists and general pediatricians should be aware of the condition as it may lead to severe complications. The present study thus describes the case of a post-term girl who was delivered at home following prolonged, obstructed labor and developed lesions on arms and limbs.

Case Report. Patient information. The newborn was delivered after a complicated pregnancy due to maternal hypertension and oligohydramnios of unknown etiology. The umbilical cord was removed at a local polyclinic. The newborn was of an unknown birth weight and Apgar score.

Clinical findings. The newborn was taken to the emergency room (ER) at the fourth day of life with abnormal movements of the upper and lower extremities, apnea, decreased oral intake, and large erythematous lesions on both arms and thighs. the newborn was treated with phenobarbitone for her seizures. She was then intubated before receiving an anti-toxoid tetanus vaccine, immunoglobulins, and antibiotics after performing a partial septic screen. She was then transferred to the pediatric intensive care unit (PICU) for hypoxemic-ischemic encephalopathy and neonatal sepsis. In the PICU, she was connected to a synchronized intermittent mechanical ventilation machine with $40 \%$ oxygen for 3 days then extubated to a non-invasive ventilator (nasal CPAP). The infant went through a complex course, which included multiorgan dysfunction, hypotension with a decreased biventricular function that required inotropes, acute kidney injury, liver dysfunction, severe hypoglycemia that required $20 \%$ dextrose infusion, and disseminated intravascular coagulopathy that required multiple blood and blood product transfusions.

Diagnostic assessment. Neurologic assessment showed increased tone in the upper and lower limbs with Earp's palsy on the left hand. A cranial ultrasound scan and electroencephalogram were normal with no epileptogenic activity or subclinical seizures. On the

Disclosure. Authors have no conflict of interests, and the work was not supported or funded by any drug company. seventh day of life, her erythematous lesions increased in size and became more nodular. The nodule was tender and warm on the buttocks, the external side of the thighs, over the dorsum of her back, and on the extensor surface of both arms and shoulders (Figures $1 \& 2$ ). These clinical symptoms were associated with normal serum calcium levels of $2.42 \mathrm{mmol} / \mathrm{L}$ (normal range: $2.2-2.62 \mathrm{mmol} / \mathrm{L}$ ) in the context of hypoalbuminemia $27 \mathrm{~g} / \mathrm{l}$ (normal range: 40.2-47.6 g/l) and high ionized calcium levels of $1.49 \mathrm{mmol} / \mathrm{L}$ (normal range: $1.17-1.32 \mathrm{mmol} / \mathrm{mL}$ ). Other laboratory analyses showed normal alkaline phosphatase level of $261 \mathrm{U} / \mathrm{L}$ (normal range: 90-270U/L), hyperphosphatemia 1.96 $\mathrm{mmol} / \mathrm{L}$ (normal range: $0.8-1.5 \mathrm{mmol} / \mathrm{L}$ ), decreased serum levels of vitamin D $13.47 \mathrm{nmol} / \mathrm{L}$ (normal range: $51-75 \mathrm{nmol} / \mathrm{L}$ ) and appropriately suppressed parathyroid hormone $4.33 \mathrm{pg} / \mathrm{mL}$ (normal range: $10-55 \mathrm{pg} / \mathrm{mL})$. Also an elevated urinary calcium $(\mathrm{mg} /$ $\mathrm{dL}$ ): urinary creatinine $(\mathrm{mg} / \mathrm{dL}$ ) ratio of 500 (in $\mathrm{mg} /$ $\mathrm{dL}: \mathrm{mg} / \mathrm{dL}$ ) (normal range: 15-20) was reported. The thyroid hormone level (TSH): $3.03 \mathrm{ul} / \mathrm{l}(\mathrm{N})$ (normal

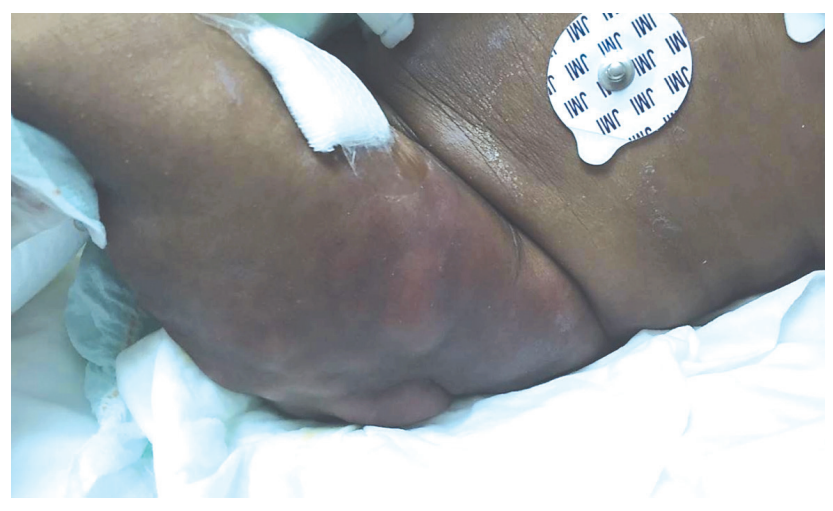

Figure 1 - Erythematous lesions on the buttocks and the lateral portions of the thighs.

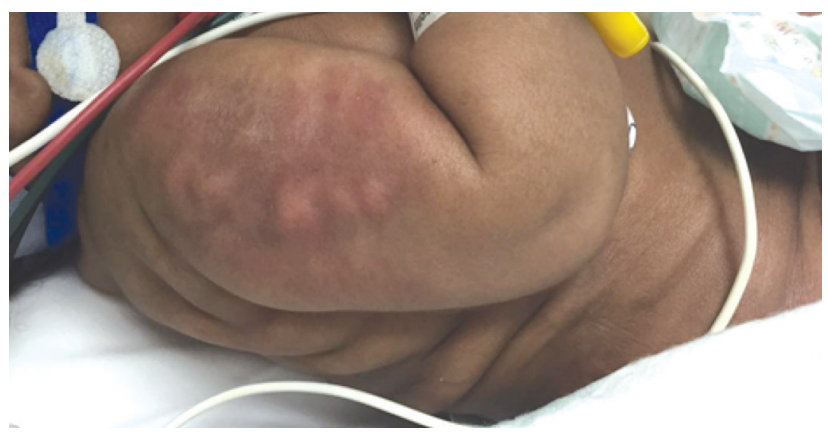

Figure 2 - Erythematous lesions were observed on the extensor surface of both arms and shoulders. 
range: $0.27-4.2 \mathrm{ul} / \mathrm{l}$ ), T3 $=8.18 \mathrm{pmol} / \mathrm{l}$ (normal range: 2.8-7 pmol/l) and T4 $=19.4 \mathrm{pmol} / \mathrm{l}$ (normal range: $12-22 \mathrm{pmol} / \mathrm{l})$. An ultrasound scan of soft tissue on the lateral side of the thigh was performed which showed a subcutaneous lesion that was hypoechogenic and homogeneous without any evidence of calcification or abscess formation. Also, the ultrasound of both right and left kidney was performed. Both the kidneys were normal in size and shape as reported by the radiologist. The length of right kidney was $4.4 \mathrm{~cm}$ and left kidney was $4.1 \mathrm{~cm}$. No hydronephrosis was observed. Mild nephrocalcinosis grade IIa in the right kidney was spotted with a slight increase at the tips of the medullary

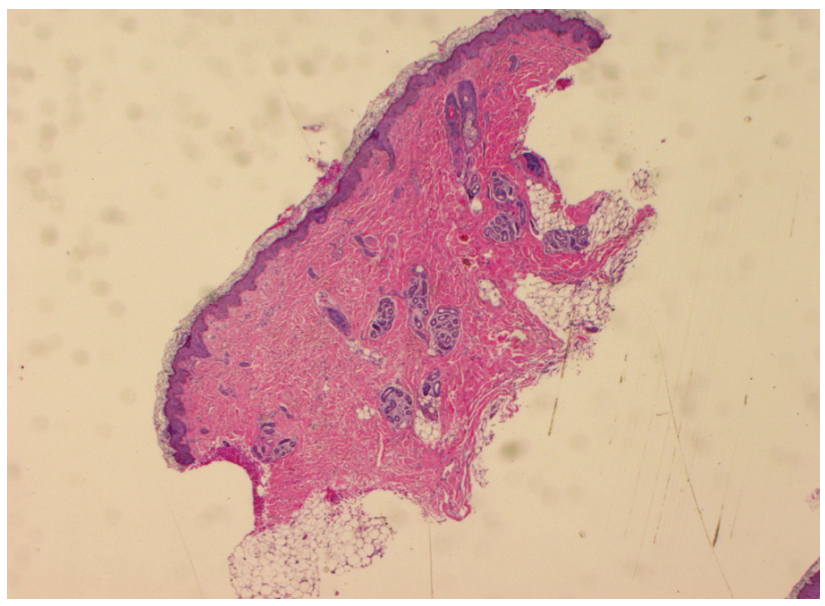

Figure 3 - Adipose tissue was mostly necrotic, exhibited residual viable foci, and contained swollen adipocytes with intracellular radial eosinophilic crystals ( $40 \mathrm{X}$ magnification used with hematoxylin and eosin (H\&E) stain) pyramids. A skin biopsy confirmed the diagnosis of SCFN and revealed marked adipose tissue that was largely necrotic. The residual viable foci exhibited swollen adipocytes with intracellular radial eosinophilic crystals (Figure 3). A summary of the patient's history and timeline of the outcomes is given in Table 1 .

Therapeutic intervention. The infant received 1.5 times of maintenance intravenous fluid for hyperhydration. Furosemide infusion was started at a rate of $1 \mathrm{mg} / \mathrm{kg} / \mathrm{h}$ and oral prednisolone was administered as $5 \mathrm{mg}$ for 20 days. The pain was controlled by morphine infusion with a cold blanket. Surgery was performed to remove the large nodules.

Follow-up and outcome. On the 11th day of life, despite aggressive treatment, her calcium levels did not show any significant improvement and serum calcium levels were still at the upper limit $(2.42 \mathrm{mmol} / \mathrm{L})$. Calcitonin was added in a dose of $16 \mathrm{U}$ and was increased to $25 \mathrm{U}$ from day 30 for 7 days under the guidance of an endocrinology team. This resulted in a decrease in her calcium level to $2.00 \mathrm{mmol} / \mathrm{L}$. Subsequently, the SCFN nodules decreased in size by 20th day of life. The serum calcium levels continued to be within the normal range during this period of treatment and the newborn was transferred to the general pediatric ward at the 30th day of life with careful monitoring by a multidisciplinary team.

Discussion. Subcutaneous fat necrosis of the newborn, is a rare type of panniculitis that has no gender predilection. It can act as a visual marker for important metabolic changes. It requires a careful, long-term treatment and follow-ups. It develops as a purplisherythematous indurated nodules and plaques within

Table 1 - Summary of the patient case from initial visit to treatment and follow-up.

\begin{tabular}{|c|c|c|c|}
\hline Dates & Summaries from initial and follow up visits & Diagnostic testing & Interventions \\
\hline $8^{\text {th }}$ March 2017 & $\begin{array}{l}\text { Four days old female, full term, home delivery } \\
\text { for unbooked mother. Had complicated and } \\
\text { prolonged delivery. Presented with signs of shock and } \\
\text { respiratory distress to the emergency department }\end{array}$ & $\begin{array}{l}\text { Partial septic screen, complete cell } \\
\text { count, liver and renal function test } \\
\text { with serum electrolytes, coagulation } \\
\text { profile }\end{array}$ & $\begin{array}{l}\text { Phenobarbitone, } \\
\text { Anti-toxoid tetanus vaccine } \\
\text { and immunoglobulins, } \\
\text { Antibiotics }\end{array}$ \\
\hline $10^{\text {th }}$ March 2017 & $\begin{array}{l}\text { Developed extensive, tender and warmerythematous } \\
\text { lesions with severe hypercalcemia }\end{array}$ & $\begin{array}{l}\text { Blood-serum testing for calcium, } \\
\text { alanine aminotransferase, } \\
\text { alkaline phosphatase, vitamin D, } \\
\text { parathyroid hormone, skin biopsy for } \\
\text { histopathology }\end{array}$ & $\begin{array}{l}\text { Hyperhydration, loop } \\
\text { diuretic, steroid, } \\
\text { analgesia (morphine) }\end{array}$ \\
\hline $12^{\text {th }}$ March 2017 & Surgery was carried out to remove large nodules & $\begin{array}{l}\text { Renal } \\
\text { Ultrasonography }\end{array}$ & Calcitonin \\
\hline $14^{\text {th }}$ March 2017 & $\begin{array}{l}\text { Patient responded to high doses of calcitonin. } \\
\text { Calcium level decreased to normal } \\
\text { Patient discharged home without complication } \\
\text { Outpatient follow up }\end{array}$ & None & None \\
\hline
\end{tabular}


the first week post-delivery. The nodules are typically located on the face, bottom, arms, thighs, and trunk ${ }^{4}$ of the newborn. However, each lesion can be of different size ranging from a few millimetres to centimetres. Nevertheless, they often resolve spontaneously during the baby's first year of life. In the present study, the newborn patient showed several distinctive features that confirmed the SCFN. Also, the skin biopsy test of the infant along with maternal complications confirmed the diagnosis. For proper analysis, the pathologist detects the patchy zones of fat necrosis enclosed by a granulomatous infiltrate of lymphocytes, macrophages, and giant cells. Adipocytes is seen as radially arranged needle-shaped clefts which are doubly refractive in polarized light microscopy. Most of the fat and multinucleate giant cells has needle-shaped clefts that mostly lie in a radial fashion. ${ }^{5}$ In the present study, the patient's histopathology specimen showed this type of arrangement confirming the diagnosis.

The major complication of subcutaneous fat necrosis (SFN) is hypercalcemia, which occurs in $25 \%$ of cases. It is due to the increased level of 1,25-dihydroxy vitamin D3, which is independent of PTH function. Changes in such hormones can occur up to 6 months after the resolution of skin lesions and can occur up to 6 months after the development of skin manifestations. ${ }^{6}$ But in the present case, the patient showed normal PTH and 1,25dihydroxy vitamin D3 levels. Persistent hypercalcemia can lead to nephrocalcinosis with subsequent chronic kidney disease, a condition which is in line with our case study.

Once the patient is diagnosed with SCFN, prolonged follow-up and aggressive treatment is vital for the patient. They often involve intravenous hyperhydration, intravenous loop diuretics, a low calcium and vitamin $\mathrm{D}$ diet, and oral corticosteroids. Calcitonin, citrate, and bisphosphonates are also effective. Hypercalcemia was initially treated via eliminating excessive calcium and vitamin $\mathrm{D}$ intake. However, adequate hydration with large amounts of intravenous saline administration is also critical as it promotes the excretion of calcium by inhibiting proximal tubular and loop sodium reabsorption. The literature reports that treatment with furosemide may help induce calciuresis, but it must be used carefully to avoid dehydration and the subsequent worsening of hypercalcemia. ${ }^{7}$ Notably, prednisone which is used to reduce the granulomatous inflammatory process and vitamin $\mathrm{D}$ production can increase urinary calcium excretion, thereby elevating a patient's risk of nephrocalcinosis. ${ }^{8}$ In the present case study, the patient was treated with vitamin D supplements following hydration and treatment with furosemide. The patient was not showing any improvement and the calcium level was not declining either. Therefore, the patient was treated with calcitonin. The calcitonin treatment decreased the level of calcium in the patient drastically. Also, this calcitonin treatment removed the need for further therapy by bisphosphonate. Subsequently, the infant's condition improved and the SCFN nodules decreased in size by the 20th day. Therefore, the present case showed that the calcitonin treatment is a good option instead of bisphosphonates therapy to maintain the calcium level in the patient.

Conventionally, SCFN goes through a self-limiting course. Therefore, skin lesion regression is expected after a few months, often without any residue on the skin. The calcium level in the blood should be checked weekly for atleast 6 months after the resolution of SCFN skin lesions in patients. However, due to the complications that SCFN manifests in later stages of life, its immediate diagnosis and treatment is highly recommended.

In conclusion, SFN is frequently diagnosed based on clinical presentation alone; however, there must be a high level of doubt among medical personnel to help early diagnosis and appropriate treatment. Ultimately, correct diagnosis leads to proper prognosis and the patient undergoes appropriate follow-up. Nevertheless, a skin biopsy can confirm the diagnosis of SFN. Moreover, the prognosis of these skin lesions is good as they usually resolve spontaneously. Follow-up after resolution of skin lesions is also important for reducing morbidity or mortality from SFN. The possible complications arising from hypercalcemia can be prevented or properly managed as well. In the case presented herein, critical hypercalcemia was unresponsive to diuretics and hyperhydration, whereas calcitonin and corticosteroids were effective for treating hypercalcemia. There were no further complications, thereby reducing the need for bisphosphonate therapy and decreasing the length of hospital stay for the patient.

Acknowledgment. We would like to thanks Editage (https:// www.editage.com/), a division of Cactus Communications for English language editing.

\section{References}

1. Rubin G, Spagnut G, Morandi F, Valerio E, Cutrone M. Subcutaneous fat necrosis of the newborn. Clin Case Rep 2015; 3: 1017-1020.

2. Chikaodinaka AA, Jude AC. Subcutaneous fat necrosis of the newborn: a case report of a term infant presenting with malaise and fever at age of 9 weeks. Case Reports in Pediatrics 2015; 638962. 
3. Gomes MP, Porro AM, Enokihara MM, Floriano MC. Subcutaneous fat necrosis of newborn: clinical manifestations in two cases. An Bras Dermatol 2013; 88: 154-157.

4. Hogeling M, Meddles K, Berk DR, et al. Extensive subcutaneous fat necrosis of the newborn associated with therapeutic hypothermia. PediatrDermatol 2012; 29: 59-63.

5. Gomes MP, Porro AM, Enokihara MM, Floriano MC. Subcutaneous fat necrosis of the newborn: clinical manifestations in two cases. An Bras Dermatol 2013; 88: 154-157.
6. Alos N, Eugène D, Fillion M, Powell J, Kokta V, Chabot G. Pamidronate: Treatment for severe hypercalcemia in neonatal subcutaneous fat necrosis. Horm Res 2006; 65: 289-294.

7. Aucharaz KS, Baker EL, Millman GC, et al. Treatment in hypercalcemia in subcutaneous fat necrosis is controversial. Horm Res 2007; 68: 31.

8. Grass B, Weibel L,Hagmann C, Brotschi B. Subcutaneous fat necrosis in neonates with hypoxic ischaemic encephalopathy registered in the Swiss National Asphyxia and Cooling Register. BMC Pediatr 2015; 9;15:73 